\title{
Guest editorial: advanced design techniques for wireless communications
}

\author{
Ana Rusu · Mohammed Ismail
}

Published online: 10 December 2008

(C) Springer Science+Business Media, LLC 2008

This special issue of the Analog Integrated Circuits and Signal Processing presents seven papers in the area of "Advanced Design Techniques for Wireless Communications". New design methodologies, EDA tools and radio transceivers architectures and topologies for emerging wireless technologies are presented. The papers presented in this issue were selected based both on the overall quality of the paper and on the timeliness of the topic.

The selected papers reflect continuing trends toward higher levels of analog and digital circuit techniques covering a wide variety of subjects within the Analog Integrated Circuits and Signal Processing fields, ranging from RF/analog and mixed-signal circuits and systems to design methodologies and tools for telecommunications applications.

In the first paper, C-Y Wu, W-C Wang, F. R Shahroury, Z-D Huang and H-J Zhan present current-mode design techniques for CMOS RF circuits, which have been developed and employed to implement a $24-\mathrm{GHz}$ CMOS receiver front-end. A receiver front-end, which comprises a current-mode LNA and a current-mode downconversion mixer, has been fabricated in $0.13-\mu \mathrm{m}$ CMOS technology. The measurement results demonstrate the feasibility of the current-mode techniques for CMOS RF circuits, showing a conversion gain of $11.3 \mathrm{~dB}$, a noise figure of $14.2 \mathrm{~dB}$, the input-referred $1-\mathrm{dB}$ compression point of $-13.5 \mathrm{dBm}$ and the input-referred third-order intercept point of $-1 \mathrm{dBm}$.

T-Y Lo, C-S Kao and C-C Hung present in their paper a CMOS 3rd order Butterworth low-pass Gm-C filter for

A. Rusu $(\bowtie) \cdot$ M. Ismail

ECS, ICT, Royal Institute of Technology (KTH), Stockholm, Sweden

e-mail: arusu@kth.se multi-mode wireless applications. A transconductor based on flipped-voltage follower circuit and an active resistor working in the strong inversion to achieve the transconductance tuning is designed for multi-mode implementations. The theoretical analysis and a complete set of measurement results demonstrate that the proposed filter is suitable for IEEE $802.11 \mathrm{a} / \mathrm{b} / \mathrm{g} / \mathrm{n}$ Wireless LANs. The filter can operate with a cutoff frequency of 10 to $20 \mathrm{MHz}$, and has a maximum power consumption of $13 \mathrm{~mW}$ from a $1.8-\mathrm{V}$ supply voltage.

The third paper, by L-F Tanguay and M. Sawan, presents the architectural choices and design of a fully integrated integer- $\mathrm{N}$ frequency synthesizer operating in the 902-928 MHz ISM band. This ultra-low power frequency synthesizer is integrated in a transceiver for implantable wireless sensing microsystems, targeting in vivo monitoring of biological parameters. The phase-locked loop-based synthesizer includes a $1.830 \mathrm{GHz}$ LC voltage controlled oscillator using a $10 \mathrm{nH}$ on chip inductor. Varactors are implemented using $\mathrm{P}+$ in $\mathrm{N}$-well diodes for their linearity and high quality factor. Several innovative design techniques have been employed to reduce the power consumption of the proposed synthesizer to $580 \mu \mathrm{W}$ under $1 \mathrm{~V}$, which is almost an order of magnitude lower compared to the recent synthesizer designs having a similar architecture.

Yi Ke, S. Radiom, J. Craninkx, G. Vandenbosch and G. Gielen present a systematic design methodology for high-order multi-bit Continuous-Time Sigma-Delta modulators. The proposed strategy provides a straightforward method for determining the coefficients of the modulator, starting with the design of the modulator in $\mathrm{z}$-domain and then converting it into the s-domain. The method is illustrated for a 4th-order 4-bit modulator with an OSR of 8 and a $20 \mathrm{MHz}$ signal bandwidth. The simulation results show 
that by applying the proposed method, the overall power consumption can be greatly reduced.

The next paper by R. Castro-López, A. Morgado, O. Guerra, R. Río, J. M de la Rosa, B. Pérez and F. V Fernández describes design techniques of reconfigurable analog-to-digital converters for multi-standard wireless communication terminals. The proposed methodology formulates a systematic design flow that ensures that efficient silicon area and power efficiency in the different operation modes are considered at all hierarchical levels. Expandable cascade modulators are considered as the starting point to further reconfigurability at the architectural level. Using a combination of accurate behavioral modeling, statistical optimization techniques, and devicelevel simulation, the proposed methodology handles the design complexity of a reconfigurable converter while ensuring adaptive power consumption and boosting hardware sharing. A case study is presented where a reconfigurable GSM/Bluetooth/UMTS modulator is designed in a $130 \mathrm{~nm}-\mathrm{CMOS}$.

E. Prefasi, L. Hernandez, S. Reekmans and P. Rombouts present in their paper a bandpass sigma-delta modulator with distributed resonators. The paper makes a brief review of the particularities of the continuous-time sigma-delta modulator architectures implemented with transmission lines and shows the experimental results of such a bandpass modulator implemented in BiCMOS technology. The measurement results show that the designed bandpass ADC converts narrow-band signals centered both at 46 and $138 \mathrm{MHz}$, respectively, with a maximum SNDR of $58 \mathrm{~dB}$, for a sampling frequency of $184 \mathrm{MHz}$ and an OSR of 256, and consumes $62 \mathrm{~mW}$.

The last paper by S. Rodriguez, J. G. Atallah, A. Rusu, L. R. Zheng and M. Ismail presents a tool capable of automatically compiling the circuit of a direct conversion receiver at the schematics level based on system specifications. The tool uses power constrained noise and linearity optimization vector-space algorithms that automatically size the transistors, passive components, and find the optimum biasing points. The solution generated by the tool is automatically read by Agilent ADS where the blocks are easily fine tuned and validated before layout. Case studies involving WiMAX, UMTS, GSM, Bluetooth and WLAN are presented to reveal the capabilities of the tool in reducing the design time.

The seven papers presented in this special issue have all undergone the standard review process of the journal. We would like to thank the reviewers for their effort and time, and for their useful comments and suggestions for improving the quality of the papers. Also, our thanks go to the authors for their positive and quick responses to the reviewers' suggestions. Finally, we would like to thank to the staff at Springer for helping us to prepare this special issue and for their efficient handling of the production of this issue.

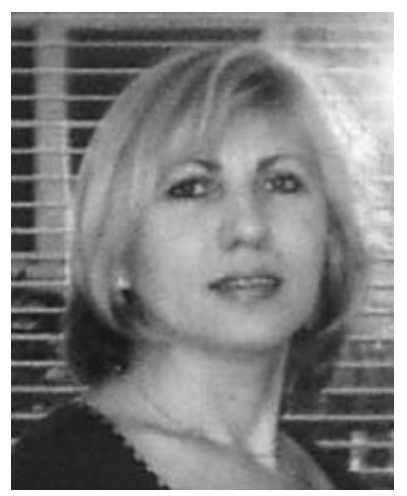

Ana Rusu (M'92) received degrees of Diploma Engineer (M.S. degree) in Electronics and Telecommunications Engineering from Technical University of Iasi, Romania, in 1983, Ph.D. in electronics from Technical University of Cluj-Napoca, Romania, in 1998 and Docent in Circuit Theory from Royal Institute of Technology Stockholm, Sweden in 2006. During 1983-1986 she was with Research Institute for Electronics and Telecommunications Bucharest, subsidiary Iasi, as a researcher. From 1986 to 1988 she was with Territorial Computer Centre, Piatra-Neamt, Romania, as a programmer in software engineering. Since 1988 she has been with the Technical University of Cluj-Napoca, Electronics and Telecommunications Faculty. In 1999 she was appointed as an associate professor. She has been in visiting researcher positions in University of Bradford, England in 1997; National Technical University of Athens, Greece in 1999; and TIMA Laboratory, Institute National Polytechnique of Grenoble, France in 2001. Since September 2001, she has been with the Royal Institute of Technology (KTH), Stockholm, Sweden, where she is a senior researcher in the RaMSiS group. Her research interests include low-voltage low-power RF/analog and mixed-signal circuits and systems design, digitally-enhanced RF/ analog/mixed-signal; EDA/CAD tools for wireless communications; Software Defined Radio technology; wireless sensor networks. She has participated in several national and international research projects and has authored or coauthored more than 80 international scientific publications in journals, books, book chapters and conference proceedings.

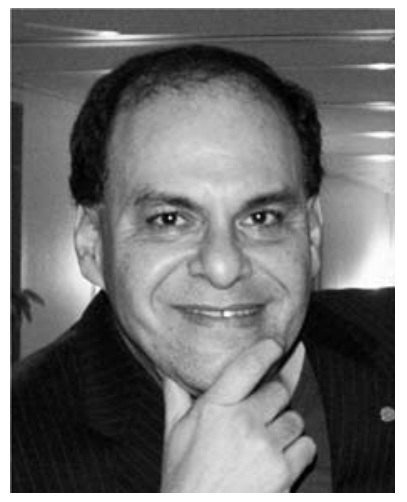

Mohammed Ismail ( $\mathrm{S}^{\prime} 80$ M'82-SM'84-F'97) has over 25 years experience of $R \& D$ in the fields of analog, RF and mixed signal integrated circuits. He has held several positions in both industry and academia and has served as a corporate consultant to nearly 30 companies in the US, Europe and the Far East. $\mathrm{He}$ is Professor of Electrical and Computer Engineering and the Founding Director of the Analog VLSI Lab at Ohio State and of the RaMSiS Group at KTH. His current interest lies in research involving digitally programmable/configurable fully integrated CMOS radios with focus on low voltage/low power "first-pass" solutions for cognitive radios, $3 \mathrm{G}$ and $4 \mathrm{G}$ wireless handhelds. He publishes intensively in this area and has been awarded 11 patents. He has co-edited and coauthored several books including a text on Analog VLSI Signal and Information Processing, (McGraw Hill). His last book (2007) is entitled Radio design in Nanometer Technologies, Springer. He advised the thesis work of $45 \mathrm{PhD}$ students and of over $85 \mathrm{MS}$ students. He co-founded ANACAD-Egypt (now part of Mentor Graphics, Inc.) and Firstpass Technologies Inc., a developer of CMOS 
radio and mixed signal IPs for handheld wireless applications. Dr. Ismail has been the recipient of several awards including the US National Science Foundation Presidential Young Investigator Award, the US Semiconductor Research Corp Inventor Recognition Awards in 1992 and 1993, The College of Engineering Lumley Research Award in 1992, 1999, 2002 and 2007 and a Fulbright/Nokia fellowship Award in 1995. He is the founder of the International Journal of Analog Integrated Circuits and Signal Processing, Springer and serves as the Journal's Editor-In-Chief. He has served as Associate Editor for many IEEE Transactions, is on the International Advisory Boards of several journals and was on the Board of Governors of the IEEE Circuits and Systems Society. He is the founder of ICECS, the CAS flagship Conference for Region 8. He obtained his BS and MS degrees in Electronics and communications from Cairo University, Egypt and the PhD degree in Electrical Engineering from the University of Manitoba, Canada. $\mathrm{He}$ is a Fellow of IEEE. 